\title{
Predictors of Adherence to a Structured Exercise Program and Physical Activity Participation in Community Dwellers after Stroke
}

\author{
Anne Tiedemann, ${ }^{1}$ Catherine Sherrington, ${ }^{1}$ Catherine M. Dean, ${ }^{2}$ Chris Rissel, ${ }^{3}$ \\ Stephen R. Lord, ${ }^{4}$ Catherine Kirkham, ${ }^{1}$ and Sandra D. O'Rourke ${ }^{4}$ \\ ${ }^{1}$ The George Institute for Global Health, The University of Sydney, Sydney, NSW 2006, Australia \\ ${ }^{2}$ Discipline of Physiotherapy, Faculty of Human Sciences, Macquarie University, North Ryde, NSW 2109, Australia \\ ${ }^{3}$ Sydney School of Public Health, The University of Sydney, Sydney, NSW 2006, Australia \\ ${ }^{4}$ Neuroscience Research Australia, The University of New South Wales, Sydney, NSW 2052, Australia
}

Correspondence should be addressed to Anne Tiedemann, atiedemann@georgeinstitute.org.au

Received 10 June 2011; Revised 18 July 2011; Accepted 27 July 2011

Academic Editor: Gillian Mead

Copyright (C) 2012 Anne Tiedemann et al. This is an open access article distributed under the Creative Commons Attribution License, which permits unrestricted use, distribution, and reproduction in any medium, provided the original work is properly cited.

Aim. To investigate predictors of adherence to group-based exercise and physical activity participation among stroke survivors. Methods. 76 stroke survivors participated (mean age 66.7 years). Adherence was the percentage of classes attended over one year. Physical activity was the average pedometer steps/day measured over seven days at the end of the trial. Possible predictors included baseline measures of demographics, health, quality of life, falls, fear of falling, cognition, and physical functioning. Results. Mean class attendance was 60\% (SD 29\%). Only one variable (slow choice stepping reaction time) was an independent predictor of higher class attendance, explaining 5\% of the variance. Participants completed an average of 4,365 steps/day (SD 3350). Those with better physical functioning (choice stepping reaction time, postural sway, maximal balance range, 10-m walk, or 6-min walk) or better quality of life (SF-12 score) took more steps. A model including SF-12, maximal balance range, and 6-min walk accounted for $33 \%$ of the variance in average steps/day. Conclusions. The results suggest that better physical functioning and health status are predictors of average steps taken per day in stroke survivors and that predicting adherence to group exercise in this group is difficult.

\section{Introduction}

Stroke is a leading cause of death and disability throughout the world [1] with 15 million people worldwide having a stroke each year [2]. The major socioeconomic burden of stroke results from the associated chronic disability rather than death [3]. For example, in Australia, there are around 350,000 stroke survivors, of whom $90 \%$ live at home and $282,000(80 \%)$ live with permanent disability [4]. People are now more likely to survive after suffering a stroke, increasing the burden of disability over the last decade. This burden will continue to increase dramatically over the next two decades as the population ages [5].

Falls are a significant contributor to stroke-related disability [6]. Over $70 \%$ of stroke survivors fall within six months of discharge from hospital [7], and nearly 50\% of community-dwelling female stroke survivors continue to fall each year [8]. Gait and balance problems have been found to be important factors underlying this increased falls risk in this group $[8,9]$.

Effective strategies to prevent falls and minimise strokerelated disability are essential to provide quality-of-life benefits and minimise spiralling health costs. There is now good evidence that well-designed exercise programs can enhance function after stroke [10-12] and can prevent falls in the general community of older people [13]. However, as is evident in the general population [14] and stroke survivors [15], ongoing adherence to exercise programs remains a major barrier to their effectiveness. An improved 
understanding of barriers to exercise adherence may assist in designing and administering optimal exercise programs.

A recent systematic review of facilitators and barriers to participation in falls prevention in older people included 24 trials [16]. The authors identified several factors associated with increased exercise participation, including high exercise self-efficacy, past exercise history, good general health, and functional independence. The specific program characteristics associated with improved adherence were frequent, moderate duration activity, program accessibility and convenience, emphasis on social aspects, strong leadership, and individually tailored exercise.

Similarly, in a recent study involving older retirement village residents [17], we identified poor balance, impaired cognition, and multiple medication usage to be significant independent predictors of poor exercise class attendance. This result indicates that the people with the poorest physical functioning were the most likely to find it difficult to adhere to the exercise program.

Walking speed and endurance among community dwelling stroke survivors are markedly lower than agematched controls $[11,12]$ and are associated with reduced quality of life and social isolation. This demonstrates the importance of physical activity promotion to stroke survivors to maximise their health outcomes. Documenting factors that predict adequate physical activity participation may also prove helpful in designing the content and delivery of exercise programs.

To address the above issues, we documented exercise class adherence and physical activity participation in a large sample of stroke survivors. The first study aim was to identify factors that were associated with adherence to a 12month supervised group-based lower-limb exercise program designed to enhance mobility and prevent falls. The second aim was to identify factors that are associated with the average number of steps taken per day at the end of the trial period. A range of health, medical, and physical status variables measured at baseline were assessed as possible predictors of adherence.

\section{Methods}

2.1. Study Design. This study comprises a secondary analysis of data from participants randomised to the lower limb intervention group of a prospective, multicentre, randomised trial [18]. The study protocol was approved by Sydney South West Area Health Service Ethics Committee (Clearance no: X060039) and The University of Sydney Human Research Ethics Committee (HREC no. 07/2006/9031), and written informed consent was obtained from all participants.

2.2. Participants. Participants were stroke club members who had suffered at least one stroke, were able to walk $10 \mathrm{~m}$ independently with or without a mobility aid, gained medical clearance for exercise, were willing to join the New South Wales Stroke Recovery Association if not already a member, were willing to commit to a weekly exercise class and home program for 12 months, and were able to give informed consent. Participants were excluded if they had a cognitive impairment defined by a Folstein Mini-Mental State Examination (MMSE) [19] score of less than 20, had insufficient communication/English skills to participate in assessment and intervention, or had a medical condition precluding exercise such as unstable cardiovascular disease or suffered from other uncontrolled chronic conditions [20] that would interfere with training and/or testing protocols. Age, side of stroke, time since stroke, and comorbidities were recorded.

2.3. Exercise Program. Study participants took part in weekly exercise classes and were given a home exercise program to be completed at least 3 times per week. Classes were conducted over 40 weeks at the Stroke Club and were provided to participants free of charge. The intensity of the exercises included in the program was progressed as performance improved to ensure the intervention remained challenging. Experienced physiotherapists specifically trained in the trial protocol delivered the exercise classes and also designed individual home programs, which were reviewed and modified monthly. Exercise sessions took between 45 and 60 minutes. The exercise intervention was designed to prevent falls, enhance mobility, and increase physical activity (the Weight-bearing Exercise for Better Balance (WEBB) program available from the authors on request). Participants were also encouraged by exercise leaders to increase the amount of walking undertaken if considered safe to do so by the leader. Further information about the intervention program can be found in the trial protocol [18].

2.4. Adherence Measures. Two measures of adherence were used to determine the amount of physical activity participation and the factors associated with greater adherence in this group. Exercise class attendance for the duration of the study was recorded by exercise leaders and then expressed as a percentage of the number of classes offered. Physical activity participation was measured by recording the number of steps taken each day for seven consecutive days using a Digimax pedometer and then calculating the average number of steps per day. This measure was recorded at the end of the 12month intervention when all classes were complete, during which time participants had been encouraged by exercise leaders to walk if safe to do so.

2.5. Predictor Variables. Data for possible predictors of exercise class attendance and averaged steps per day were collected via an interview, and a physical assessment was conducted at baseline by a physiotherapist. Potential predictor variables were grouped in the following domains: demographic, health and health-related quality of life, falls and fear of falling, cognition, muscle strength, standing balance, and mobility.

Demographic data (age, gender) were collected during the baseline questionnaire. Health was measured by asking about the presence of or history of a number of health 
conditions and symptoms (visual problems, hearing problems, Parkinson's disease, peripheral vascular disease, diabetes, heart conditions, hypertension, asthma, incontinence, epilepsy, osteoporosis, arthritis, hip fracture, vertigo, and pain). Health-related quality of life was measured using the SF12 Version 2 [21]. Possible predictor variables from the health and health-related quality-of-life domain used in the analyses were the total number of health conditions or symptoms, incontinence, poor vision (as measured in the physical assessment on the Melbourne Edge test of visual contrast sensitivity), the SF12 Version 2 physical composite, and SF12 Version 2 mental composite scores (0-100) [22]. Falls were assessed with a yes/no question about whether the person had experienced recurrent falls. Fear of falling was measured with a single question; "Are you afraid of falling?" which required a "yes" or "no" response. Cognition was measured using the Montreal Cognitive Assessment [23]. The Montreal Cognitive Assessment assesses different cognitive domains: attention and concentration; executive function; memory; language; visuoconstructional skills; conceptual thinking; calculations; orientation. The Montreal Cognitive Assessment is scored from 0 to 30 points.

The domains of muscle strength, standing balance, and mobility were assessed by trained physiotherapists using performance-based tests. Muscle strength was evaluated by measuring knee extensor strength in both legs in a seated position. Strength $(\mathrm{kg})$ was recorded as the best score from three attempts for each leg. Two aspects of mobility, walking speed, and capacity were assessed. Walking speed $(\mathrm{m} / \mathrm{s})$ was measured using the 10- $m$ walk test. Participants were timed as they walked at their comfortable and fastest speed over the middle $10 \mathrm{~m}$ of a level $14 \mathrm{~m}$ walking track. Walking capacity was measured by quantifying the distance walked (m) during a 6-min walk test [24]. Standing balance was assessed using three tests involving different movements in standing. The maximal balance range [25] test measures the maximal forward- and backward-leaning capacity of the participant. Subjects were asked to lean forward as far as possible from the ankles without moving the feet, then back as far as possible. Maximal anterior-posterior distance moved was measured in millimetres with a sway meter that measured displacements of the body at the level of the pelvis. For the maximal balance range test the sway meter extended in the anterior plane. Participants had three attempts at the test, with the best trial used in the analysis. Postural sway [26] was also assessed using a sway meter, positioned around the waist and extending posteriorly. Testing was performed with participants standing with eyes open on the floor and on a foam rubber mat ( $40 \mathrm{~cm}$ by $40 \mathrm{~cm}$ by $7.5 \mathrm{~cm}$ thick) [26]. Choice stepping reaction time was measured as the time(s) to complete a standardized stepping routine, where participants were required to step from either leg onto targets on the floor in response to a verbal cue directing the foot placement [27]. This is a modified version of an electronically timed test previously found to be a good composite measure of the risk of falling [27].

2.6. Statistical Analyses. Analyses were conducted using the SPSS and Stata software packages. Linear regression was used to determine the univariate associations between potential predictor variables and (a) exercise class attendance and (b) average pedometer steps per day. Predictor variables for which the individual $P$ values were less than 0.2 were then included as candidate predictor variables for multivariate linear regression models. To ensure there were at least 15 cases for each predictor [28], one potential predictor variable from each domain with a maximum of $4-5$ variables was included in each of the multivariate linear regression models. Where there was more than one variable from each domain that was associated with the outcome in univariate analyses, the variable with the lowest $P$ value was entered. Statistical assumptions underlying linear regression models were met in the final models for adherence and steps taken. Specifically, the residuals were normally distributed, and there was no indication that the residuals were heteroscedastic and no indication of a nonlinear relationship between predictor and outcome variables. There was also no indication of multicollinearity as the variables were not highly correlated $(r<0.5)$, and the variance inflation factor values for each variable were less than 10 .

\section{Results}

One hundred and fifty-one participants were recruited to the RCT investigating the effects of exercise on falls and mobility. These people were recruited from 11 stroke clubs between November 2006 and January 2009. Seventy-six participants ( 38 male and 38 female) were allocated to the lower limb exercise group, and these people are included in the analyses described here. The mean age of the participants was 66.7 years (SD 14.3), and average time since stroke was 6.7 years (SD 6.7; range 0.1-24.8). The baseline characteristics of the sample are shown in Table 1.

3.1. Exercise Class Adherence. An average of 31 (SD 8) exercise classes were available to participants over the 12month study period. Average attendance rates were $60 \%$ (SD $29 \%$ ). Eight physiotherapists, on average 28 (SD 13) years since graduating, provided the intervention at the 11 stroke clubs.

Univariate linear regression analyses identified just one variable to be significantly $(P<0.05)$ associated with better exercise class attendance; slower choice stepping reaction time $(P=0.03$, coefficient $0.2,95 \%$ CI 0.02 to 0.4$)$. Those with more comorbidities $(P=0.08)$, a self-reported fear of falling $(P=0.21)$, or a history of recurrent falls $(P=0.20)$ were also more likely to attend more classes, but these relationships did not reach statistical significance. The variables included in the analysis but not found to be predictors of class attendance included age, gender, vision, incontinence, SF-12 mental composite scores, cognition, knee extensor muscle strength, maximal balance range, postural sway, 6-min walk test distance, and $10-\mathrm{m}$ walking speed. The results of the univariate analyses are included in Table 2.

The three variables with individual $P$ values from the univariate analyses that were less than 0.2 and which 
TABLE 1: Characteristics of participants on entry to study.

\begin{tabular}{|c|c|}
\hline Characteristic & $\begin{array}{l}\text { Lower limb exercise } \\
\text { group }(n=76)\end{array}$ \\
\hline \multicolumn{2}{|l|}{ Demographics } \\
\hline $\begin{array}{l}\text { Age }(y r) \\
\text { mean }(\mathrm{SD} ; \text { range })\end{array}$ & $66.7(14.3 ; 31-91)$ \\
\hline $\begin{array}{l}\text { Time since stroke at enrolment }(y r) \text {, } \\
\text { mean (SD; range) }\end{array}$ & $6.7(6.7 ; 0.1-24.8)$ \\
\hline $\begin{array}{l}\text { Mini-Mental State } \\
\text { Examination score }(0-30) \\
\text { mean }(\mathrm{SD} \text {; range) }\end{array}$ & $27(3 ; 20-30)$ \\
\hline Gender, $n$ males (\%) & $38(50)$ \\
\hline Side of hemiplegia, $n$ right (\%) & $34(45)$ \\
\hline Recurrent falls, $n(\%)$ & $19(25)$ \\
\hline Fear of falling, $n(\%)$ & $34(45)$ \\
\hline \multicolumn{2}{|l|}{ Health conditions/symptoms } \\
\hline Visual impairment, $n(\%)$ & $61(80)$ \\
\hline Hearing impairment, $n(\%)$ & $26(34)$ \\
\hline Parkinson’s Disease, $n(\%)$ & $1(1)$ \\
\hline PVD/Leg ulcers, $n(\%)$ & $4(5)$ \\
\hline Diabetes, $n(\%)$ & $16(21)$ \\
\hline Heart condition, $n(\%)$ & $26(34)$ \\
\hline Hypertension, $n(\%)$ & $44(58)$ \\
\hline Asthma/COPD, $n(\%)$ & $16(21)$ \\
\hline Incontinence, $n(\%)$ & $20(26)$ \\
\hline Epilepsy, $n(\%)$ & $14(18)$ \\
\hline Osteoporosis, $n(\%)$ & $16(21)$ \\
\hline Arthritis, $n(\%)$ & $34(45)$ \\
\hline Hip fracture, $n(\%)$ & $5(7)$ \\
\hline Vertigo/dizziness, $n(\%)$ & $29(38)$ \\
\hline Pain, $n(\%)$ & $47(62)$ \\
\hline
\end{tabular}

represented different predictor domains were entered into a multivariate regression model (number of medical conditions and symptoms, history of recurrent falls, and choice stepping reaction time). This model explained $8 \%$ of the variability in exercise class attendance. As shown in Table 3, none of the individual variables were statistically significant in the multivariate model (although $P=0.055$ for choice stepping reaction time) indicating some shared explanatory ability. The adjusted $r^{2}$ of a model including choice stepping reaction time only was $5 \%$, indicating a small amount of additional predictive ability of the other variables and a high proportion of unexplained variability.

3.2. Physical Activity Participation. Averaged steps per day data were available for 64 participants. Overall, the mean number of steps recorded was 4,365 steps per day (SD 3,350 ), and the range was 55 to 12733 steps. This mean represents $44 \%$ of the 10,000 steps recommended by physical activity guidelines to improve health and wellbeing. In the univariate analyses, several variables were significantly associated $(P<0.05)$ with averaged steps per day. These included a better SF12v2 physical composite score and each of the balance and mobility measures (better postural sway while standing on the foam mat, better maximal balance range, better choice stepping reaction time, better 6-min walk test distance, and better 10-m walk test time). There was no association between steps recorded and age, gender, vision, incontinence, total number of health conditions or symptoms, SF12v2 mental composite score, fear of falling, recurrent falls, cognition, or leg muscle strength. The results of the univariate analyses are included in Table 2.

Three of these significant variables representing one variable from each of the domains of health/health-related quality of life (SF12v2 physical composite score), standing balance (maximal balance range), and mobility (6-min walk test) were entered into a multivariate regression model to determine independent predictors of averaged steps per day. The univariate $P$ values for maximal balance range and postural sway were both less than 0.001 . The maximal balance range measure explained slightly more variability in averaged steps per day (adjusted $r^{2} 15.9 \%$ versus $15.7 \%$ ), so this measure was chosen for the multivariate model. Similarly, the $P$ values for 6-min walk test and 10-metre walk distance were both less than 0.001 , but the 6 -min walk test explained more of the variability (adjusted $r^{2} 34 \%$ versus $27 \%$ ). The 3 -variable model explained $33 \%$ of the variability, and, as shown in Table 3, better 6-min walk test performance remained strongly associated with averaged steps per day $(P<0.001)$ in the multivariate model. Thus, for predicting averaged steps per day, we found no benefit from assessing these other variables in addition to the 6-min walk test. There was also no indication of multicollinearity as the variables were not highly correlated $(r<0.5)$ and the variance inflation factor values for each variable were less than 10.

\section{Discussion}

Our community dwelling sample of older people had been living with stroke on average for more than five years. They walked at three quarters the speed [29] and covered half the distance in six minutes compared to that of healthy older people [30] and took approximately $44 \%$ of the recommended 10,000 steps/day [31].

Exercise class attendance rates for the group ranged from 0 to $100 \%$ with a mean of $60 \%$ (SD 29\%). Contrary to expectation, we found that exercise class attendance was better among those with poorer physical functioning. This indicates that it is possible to design and deliver exercise classes that are acceptable to stroke survivors with impaired physical abilities. Choice stepping reaction time was the baseline measure of physical performance that best predicted attendance over the 12-month follow-up period. None of the other physical performance variables measured at baseline were associated with class attendance. There was some additional explanatory power of measures of comorbidity and recurrent falls that also indicated that those who were more impaired attended more exercise classes.

At baseline, we assessed demographic, health, health related quality of life, cognition, and physical performance using a variety of measures. Despite this, the variables we 


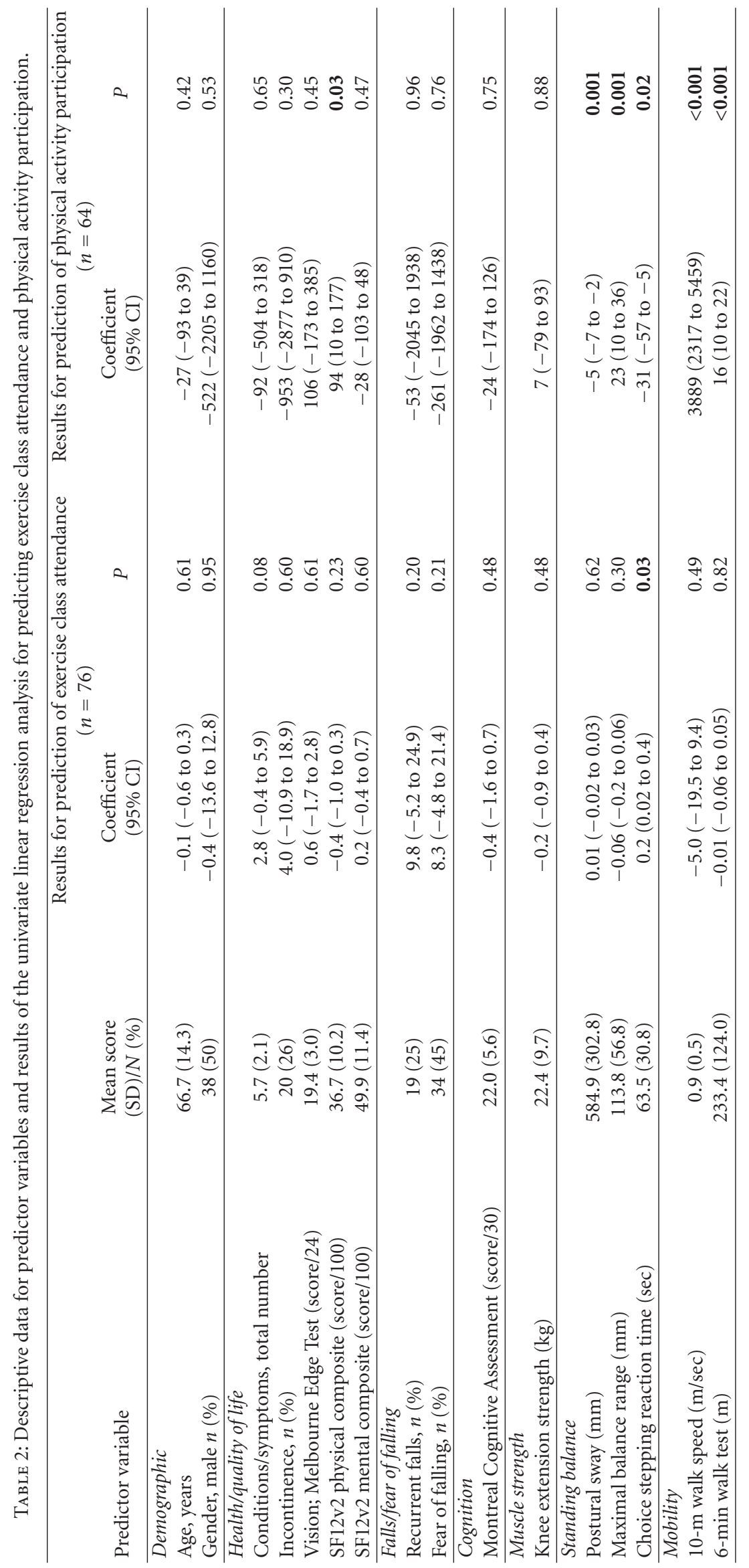


TABLE 3: Results of the multivariate linear regression analysis for predicting exercise class attendance and physical activity participation.

\begin{tabular}{|c|c|c|c|}
\hline \multirow[b]{2}{*}{ Predictor variable } & \multicolumn{3}{|c|}{ Results for prediction of exercise class attendance } \\
\hline & Coefficient $(95 \%$ CI $)$ & $P$ & Adjusted $R^{2}(\%)$ \\
\hline Conditions/symptoms (total number) & $1.99(-1.02$ to 5.00$)$ & 0.191 & 8 \\
\hline Fear of falling (yes/no) & $7.50(-4.93$ to 19.93$)$ & 0.233 & \\
\hline \multirow[t]{2}{*}{ Choice stepping reaction time (sec) } & $0.20(-0.004$ to 0.40$)$ & 0.055 & \\
\hline & \multicolumn{3}{|c|}{ Results for prediction of physical activity participation } \\
\hline SF12v2 physical composite (score/100) & $11.60(-65.62$ to 88.80$)$ & 0.765 & 33 \\
\hline Maximal balance range $(\mathrm{mm})$ & $7.77(-6.05$ to 21.6$)$ & 0.265 & \\
\hline 6-min walk test $(\mathrm{m})$ & $13.60(6.58$ to 20.61$)$ & 0.000 & \\
\hline
\end{tabular}

measured were not able to explain the majority of the variability $(92 \%)$ in class attendance rates over the 12 month follow-up period. Either the measurement tools we used were not able to accurately measure these factors in this population, or these factors are not important in the prediction of exercise adherence in stroke survivors. Factors such as depression, family support, and social isolation were not measured in our study but have been previously found to be associated with uptake and adherence with exercise programs in stroke survivors [15]. There was no cost to take part in the study, and none of the participants were in paid employment; therefore, these factors were not barriers to participation.

Our study involved the establishment of exercise classes within stroke clubs where stroke survivors were already meeting regularly. Our results may not generalise to the broader population of community-dwelling stroke survivors as our participants may have been different from stroke survivors who do not choose to meet regularly with their peers. Furthermore, most of the study participants relied on community transport or transport assistance from a relative or friend to travel to the exercise classes, so it is likely that some missed classes were due to the occasional unavailability of transport.

Physical activity participation, as measured by the average number of steps taken per day after a 12-month intervention period, was predicted by baseline performance in several tests of balance and mobility and health-related quality of life (SF-12 physical composite scale). The 6-min walk test was the strongest predictor of physical activity participation, but the measure of mobility (the 10-m walk test) was also a very strong predictor and may be easier to measure. While pedometers are commonly used as a motivational tool in patient behaviour change programs, they do undercount steps for those people with gait impairment [32]. This undercounting is likely to strengthen the association between walking speed and physical activity (step counts). Although several balance and mobility variables were associated with averaged steps per day in the univariate analyses, they did not remain significantly associated with averaged steps per day in the multivariate model which included the 6-min walk test. These variables were moderately correlated $(r=0.43-0.89)$ which indicates they are likely to be measuring similar factors. Additionally, exercise class attendance in itself was not a predictor of independent physical activity participation $(P=0.535)$.
It therefore appears that physical activity among stroke survivors was influenced by mobility but not the other study variables included here as possible predictors. This result suggests that advice and guidance to participate in regular physical activity should be targeted at all stroke survivors, regardless of individual comorbidities and functional status. In addition, people with impaired mobility may need more specific advice and different strategies to increase their physical activity levels as their steps per day may be limited by mobility. Cycling and arm cranking have been previously suggested as ways to increase physical activity in people with impaired mobility $[33,34]$. Of course, exercise prescription should consider individual impairments (e.g., arm cranking may be difficult for those with affected upper limbs), may need modified equipment, and should follow preexercise screening protocols [20] to ensure safety.

Averaged steps per day data were missing for 12 people due to difficulty using the equipment for one participant and loss to 12-month follow-up for 11 others. It is possible that this resulted in an overestimation of physical activity participation as more than half of those people lost to followup cited health problems as the reason for nonattendance at the 12-month reassessment. Furthermore, formal accuracy testing of the pedometers with all participants was not carried out prior to their use, which may have led to inaccuracies in the measures obtained.

The predictor variables we measured at baseline explained a greater proportion of the variability in averaged steps per day (33\%) compared with exercise class attendance $(8 \%)$. Although this finding should be replicated in other populations of stroke survivors, it suggests that physical activity can be more easily predicted than exercise class attendance.

\section{Conclusions}

The uptake and sustained compliance with recommendations around the importance of ongoing, regular physical activity for prevention of disease and maintenance of health is as important in stroke survivors as in the general population. The results of this study confirm that participation in specific structured exercise programs and general physical activity is difficult for many long-term stroke survivors. We found exercise class attendance was variable and difficult to predict but better among those with poorer mobility at 
baseline. Conversely, we found a higher average number of steps taken per day among those with better mobility. We hope that these results assist in the design of physical activity programs for stroke survivors.

\section{Conflict of Interests}

Professor S. R. Lord developed the The Physiological Profile Assessment (FallScreen) which is commercially available through Neuroscience Research Australia.

\section{Acknowledgments}

The research team acknowledges the contribution of Michelle Sharkey, Professor Robert Cumming, and Dr. Ruth Barker to the original trial. The research team also acknowledges contributions from Sydney South West Area Health Service Health Promotion Unit; The Stroke Recovery Association of NSW; physiotherapists involved in the study; NSW Health and University of Sydney. The project was funded by NSW Health through the NSW Health Promotion Demonstration Research Grants Scheme and conducted by the University of Sydney, Stroke Recovery Association of NSW, and Health Promotion Service of Sydney South West Area Health Service.

\section{References}

[1] A. Di Carlo, "Human and economic burden of stroke," Age and Ageing, vol. 38, no. 1, pp. 4-5, 2009.

[2] J. Mackay and G. Mensah, Eds., The Atlas of Heart Disease and Stroke, World Health Organization, Geneva, Switzerland, 2004.

[3] S. Senes, "How we manage stroke in Australia," Tech. Rep. Cvd 31, Australian Institute of Health and Welfare, Canberra, Australia, 2006.

[4] C. D. A. Wolfe, "The impact of stroke," British Medical Bulletin, vol. 56, no. 2, pp. 275-286, 2000.

[5] M. Menken, T. L. Munsat, and J. F. Toole, "The global burden of disease study: implications for neurology," Archives of Neurology, vol. 57, no. 3, pp. 418-420, 2000.

[6] National Institute for Clinical Excellence, Clinical Practice Guideline for the Assessment and Prevention of Falls in Older People, National Institute for Clinical Excellence, Uk, 2004.

[7] A. Forster and J. Young, "Incidence and consequences of falls due to stroke: a systematic inquiry," British Medical Journal, vol. 311, no. 6997, pp. 83-86, 1995.

[8] S. E. Lamb, L. Ferrucci, S. Volapto, L. P. Fried, and J. M. Guralnik, "Risk factors for falling in home-dwelling older women with stroke: the women's health and aging study," Stroke, vol. 34, no. 2, pp. 494-500, 2003.

[9] H. Stolze, S. Klebe, C. Zechlin, C. Baecker, L. Friege, and G. Deuschl, "Falls in frequent neurological diseases: prevalence, risk factors and aetiology," Journal of Neurology, vol. 251, no. 1, pp. 79-84, 2004.

[10] Outpatient Service Trialists, "Rehabilitation therapy services for stroke patients living at home: systematic review of randomised trials," The Lancet, vol. 363, no. 9406, pp. 352356, 2004.

[11] C. M. Dean, C. L. Richards, and F. Malouin, "Task-related circuit training improves performance of locomotor tasks in chronic stroke: a randomized, controlled pilot trial," Archives of Physical Medicine and Rehabilitation, vol. 81, no. 4, pp. 409417, 2000.

[12] L. Ada, C. M. Dean, J. M. Hall, J. Bampton, and S. Crompton, "A treadmill and overground walking program improves walking in persons residing in the community after stroke: a placebo-controlled, randomized trial," Archives of Physical Medicine and Rehabilitation, vol. 84, no. 10, pp. 1486-1491, 2003.

[13] L. D. Gillespie, M. C. Robertson, W. J. Gillespie et al., "Interventions for preventing falls in older people living in the community," Cochrane Database of Systematic Reviews, no. 2, Article ID CD007146, 2009.

[14] Australian Institute of Health and Welfare, "Australia's health 2010," Tech. Rep. 12, Canberra, Australia, 2010, Australia's health series.

[15] J. H. Morris and B. Williams, "Optimising long-term participation in physical activities after stroke: exploring new ways of working for physiotherapists," Physiotherapy, vol. 95, no. 3, pp. 227-233, 2009.

[16] F. Bunn, A. Dickinson, E. Barnett-Page, E. Mcinnes, and K. Horton, "A systematic review of older people's perceptions of facilitators and barriers to participation in falls-prevention interventions," Ageing and Society, vol. 28, no. 4, pp. 449-472, 2008.

[17] A. Tiedemann, C. Sherrington, and S. R. Lord, "Predictors of exercise adherence in older people living in retirement villages," Preventive Medicine, vol. 52, no. 6, pp. 480-481, 2011.

[18] C. M. Dean, C. Rissel, M. Sharkey et al., "Exercise intervention to prevent falls and enhance mobility in community dwellers after stroke: a protocol for a randomised controlled trial," BMC Neurology, vol. 9, p. 38, 2009.

[19] M. F. Folstein, S. E. Folstein, and P. R. McHugh, "Mini mental state. A practical method for grading the cognitive state of patients for the clinician," Journal of Psychiatric Research, vol. 12, no. 3, pp. 189-198, 1975.

[20] ACSM: American college of sports medicine position stand, "Exercise and physical activity for older adults," Medicine and Science in Sports and Exercise, vol. 30, no. 6, pp. 992-1008, 1998.

[21] J. E. Ware, M. Kosinski, and S. D. Keller, "A 12-item short-form health survey: construction of scales and preliminary tests of reliability and validity," Medical Care, vol. 34, no. 3, pp. 220233, 1996.

[22] J. E. Ware, M. Kosinski, and S. D. Keller, Sf-12: How to Score the sf-12 Physical and Mental Health Summary Scales, Quality Metric Incorporated, Lincoln, RI, USA, 3rd edition, 1998.

[23] Z. S. Nasreddine, N. A. Phillips, V. Bédirian et al., "The montreal cognitive assessment, MoCA: a brief screening tool for mild cognitive impairment," Journal of the American Geriatrics Society, vol. 53, no. 4, pp. 695-699, 2005.

[24] D. P. Lipkin, A. J. Scriven, T. Crake, and P. A. Poole-Wilson, "Six minute walking test for assessing exercise capacity in chronic heart failure," British Medical Journal, vol. 292, no. 6521, pp. 633-635, 1986.

[25] S. R. Lord, J. A. Ward, and P. Williams, "Exercise effect on dynamic stability in older women: a randomized controlled trial," Archives of Physical Medicine and Rehabilitation, vol. 77, no. 3, pp. 232-236, 1996.

[26] S. R. Lord, H. B. Menz, and A. Tiedemann, "A physiological profile approach to falls risk assessment and prevention," Physical Therapy, vol. 83, no. 3, pp. 237-252, 2003.

[27] S. R. Lord and R. C. Fitzpatrick, "Choice stepping reaction time: a composite measure of falls risk in older people," 
Journals of Gerontology — Series A, vol. 56, no. 10, pp. M627M632, 2001.

[28] P. Peduzzi, J. Concato, E. Kemper, T. R. Holford, and A. R. Feinstem, "A simulation study of the number of events per variable in logistic regression analysis," Journal of Clinical Epidemiology, vol. 49, no. 12, pp. 1373-1379, 1996.

[29] R. W. Bohannon, "Comfortable and maximum walking speed of adults aged 20-79 years: reference values and determinants," Age and Ageing, vol. 26, no. 1, pp. 15-19, 1997.

[30] P. L. Enrichi and D. L. Sherrill, "Reference equations for the six-minute walk in healthy adults," American Journal of Respiratory and Critical Care Medicine, vol. 158, no. 5, pp. 1384-1387, 1998.

[31] C. Tudor-Locke and D. R. Bassett, "How many steps/day are enough? preliminary pedometer indices for public health," Sports Medicine, vol. 34, no. 1, pp. 1-8, 2004.

[32] E. V. Cyarto, A. M. Myers, and C. Tudor-Locke, "Pedometer accuracy in nursing home and community-dwelling older adults," Medicine and Science in Sports and Exercise, vol. 36, no. 2, pp. 205-209, 2004.

[33] M. J. Lee, S. L. Kilbreath, M. F. Singh et al., "Comparison of effect of aerobic cycle training and progressive resistance training on walking ability after stroke: a randomized sham exercise-controlled study," Journal of the American Geriatrics Society, vol. 56, no. 6, pp. 976-985, 2008.

[34] I. Zwierska, R. D. Walker, S. A. Choksy, J. S. Male, A. G. Pockley, and J. M. Saxton, "Upper- vs lower-limb aerobic exercise rehabilitation in patients with symptomatic peripheral arterial disease: a randomized controlled trial," Journal of Vascular Surgery, vol. 42, no. 6, pp. 1122-1130, 2005. 


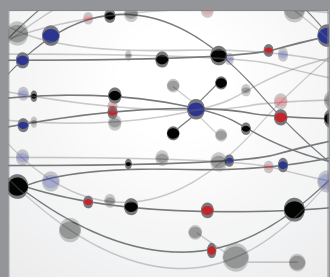

The Scientific World Journal
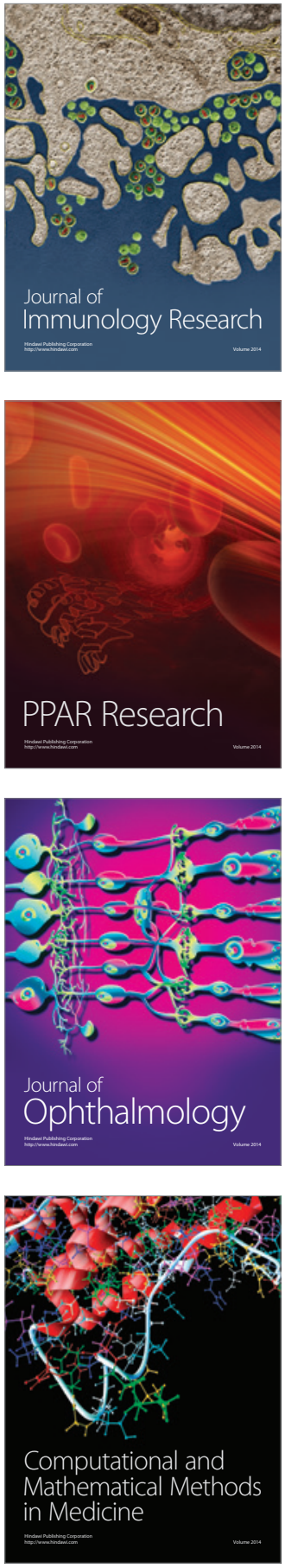

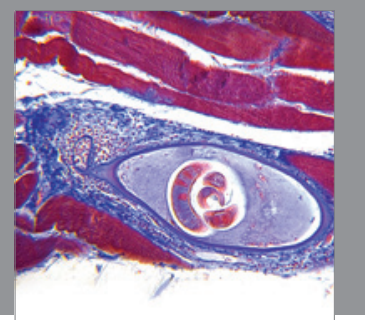

Gastroenterology

Research and Practice
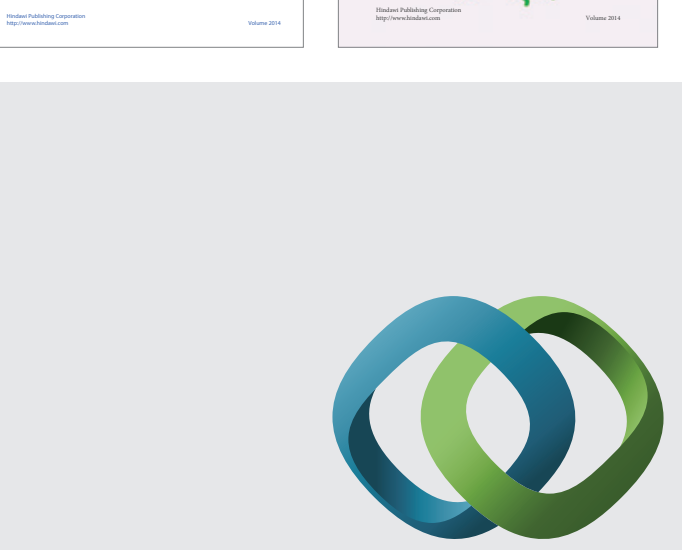

\section{Hindawi}

Submit your manuscripts at

http://www.hindawi.com
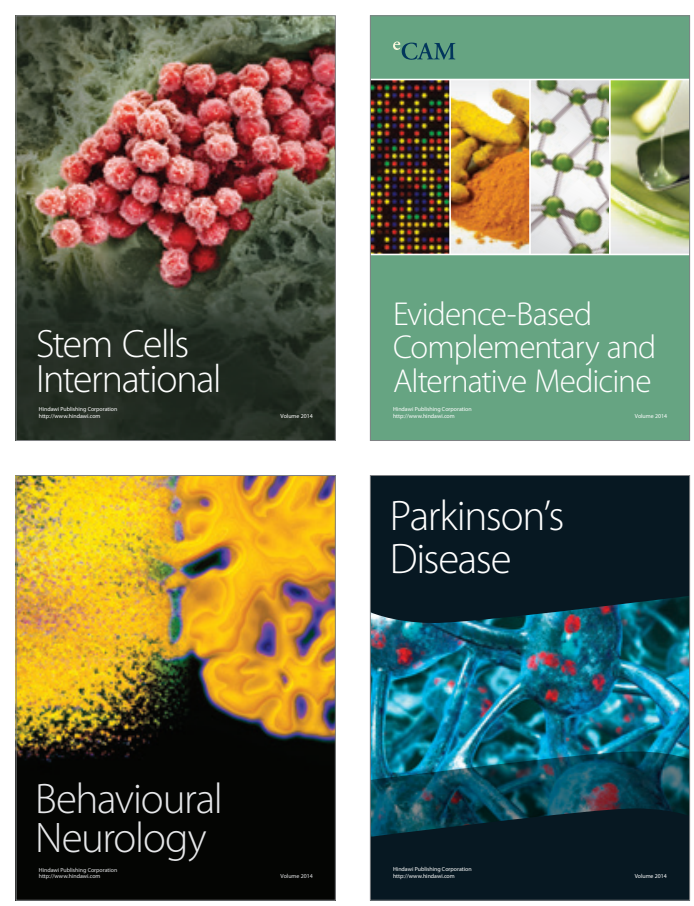

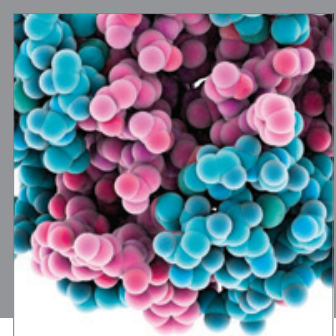

Journal of
Diabetes Research

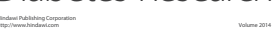

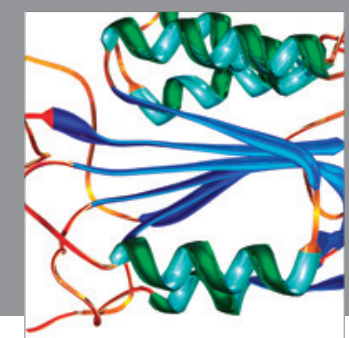

Disease Markers
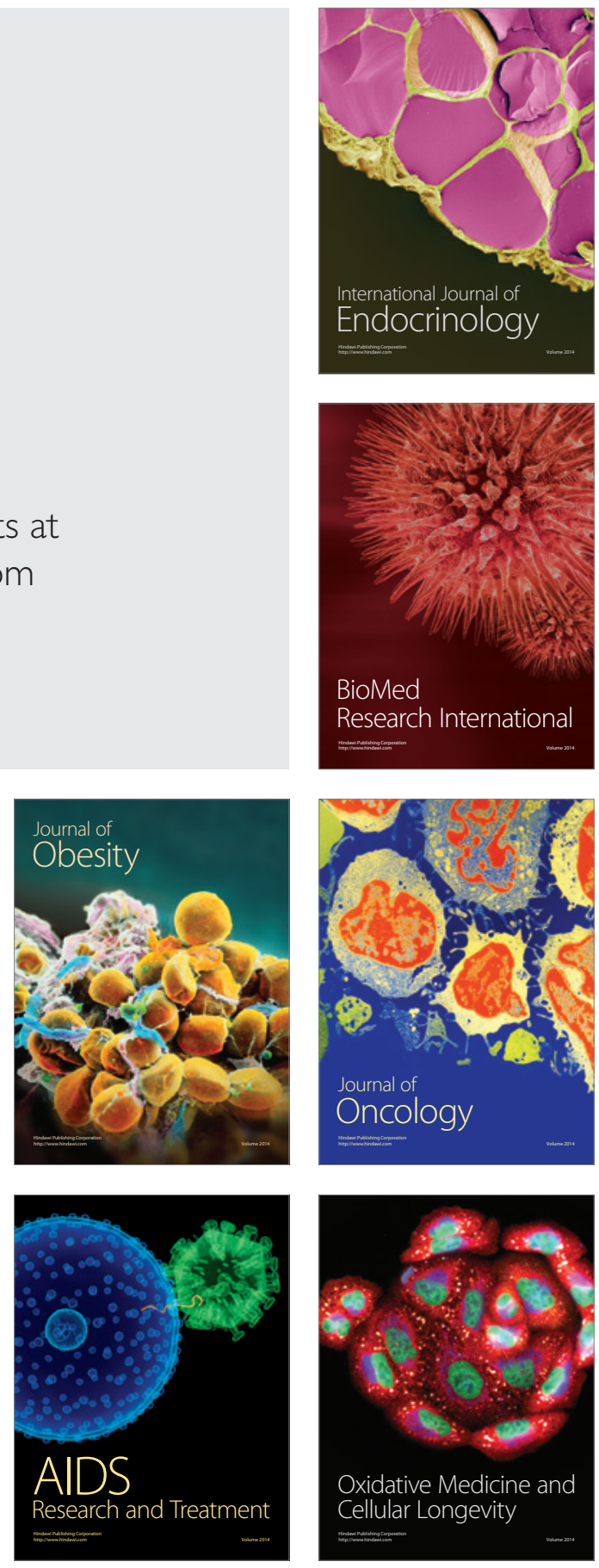